\title{
From 2-Dimensional Surfaces to Cosmological Solutions
}

\author{
A. Szereszewski and J. Tafel \\ Institute of Theoretical Physics, University of Warsaw, Hoża 69, 00-681 War- \\ saw, Poland, email: tafel@fuw.edu.pl
}

\begin{abstract}
.
We construct perfect fluid metrics corresponding to spacelike surfaces invariant under a 1-dimensional group of isometries in 3-dimensional Minkowski space. Under additional assumptions we obtain new cosmological solutions of Bianchi type $I I, V I_{0}$ and $V I I_{0}$. The solutions depend on an arbitrary function of time, which can be specified in order to satisfy an equation of state.
\end{abstract}

PACS number: 04.40.Nr

Keywords: Einstein equations, cosmological solutions, surfaces

\section{Introduction}

Perfect fluid solutions of the Einstein equations with a 2-dimensional group of isometries play an important role in nonhomogeneous cosmology [1]. Usually they are obtained under additional assumptions as separability of the metric coefficients, degeneracy of the Weyl tensor or existence of conformal symmetries (see [2] and references therein). Some of these solutions are free of singularities [3] [4] [5].

In [6] we presented a geometric construction of $G_{2}$ perfect fluid metrics for which the Einstein equations reduce to a single differential equation. The metrics are related to 2-dimensional surfaces in 3-dimensional nonphysical Minkowski space $M^{2,1}$. In this paper we apply this method to surfaces which 
are invariant under a 1-dimensional group of isometries of $M^{2,1}$. Under additional assumptions the corresponding metrics admit 3-dimensional groups of isometries. In this way we obtain tilted or nontilted cosmological solutions of Bianchi type $I I, V I_{0}$ or $V I I_{0}$. They are given up to the differential equation. We solve this equation in quadratures in the case of vanishing tilt. The resulting metrics depend on a free function. Hence, a barotropic equation of state can be imposed, however this probably requires numerical calculations. Up to knowledge of the authors the presented solutions are new [2].

For a completeness we describe below the basic construction of [6]. Let $M^{2,1}$ be the 3 -dimensional Minkowski space with coordinates $x^{i}, i=1,2,3$, and the metric $\eta_{i j}=\operatorname{diag}(1,1,-1)$. Consider a spacelike surface $\Sigma \subset M^{2,1}$ with the negative Gauss curvature $K$

$$
K<0
$$

The internal coordinates of $\Sigma$ are denoted by $x^{A}, A=0,1$. Let $\vec{n}=\left(n_{i}\right)$ be the normal vector of $\Sigma$ such that

$$
\vec{n}^{2}=-1, n_{3}<0,
$$

where $\vec{n}^{2}=n_{1}^{2}+n_{2}^{2}-n_{3}^{2}$. We relate with $\Sigma$ the following 4-dimensional spacetime metric

$$
g=\gamma e^{\psi} g_{A B}^{\prime} d x^{A} d x^{B}+\rho n_{a b} d x^{a} d x^{b}
$$

where $\gamma= \pm 1, g_{A B}^{\prime}$ is a fixed metric proportional to the second fundamental form of $\Sigma$, functions $x^{a}, a=2,3$, together with $x^{A}$ compose a 4-dimensional system of coordinates and

$$
\begin{gathered}
\rho=|K|^{-1 / 2}, \\
n_{a b}=\left(\begin{array}{cc}
n_{3}+n_{1} & n_{2} \\
n_{2} & n_{3}-n_{1}
\end{array}\right) .
\end{gathered}
$$

Components of $g$ are independent of the coordinates $x^{a}$. Hence, $\partial_{a}$ are the Killing vector fields.

Consider the following equation

$$
\operatorname{det}\left(\tilde{R}_{A B}\right)=0
$$

where

$$
\begin{gathered}
\tilde{R}_{A B}=R_{A B}+\frac{\square \rho}{2 \rho} g_{A B}, \\
R_{A B}=\frac{1}{2} R^{(2)} g_{A B}-\frac{1}{\rho} \rho_{\mid A B}+\frac{1}{2 \rho^{2}} \rho_{, A} \rho_{, B}-\frac{1}{2} \vec{n}_{, A} \vec{n}_{, B} .
\end{gathered}
$$


Here the symbol ${ }_{\mid A}$ denotes the covariant derivative with respect to the metric $g_{A B}=\gamma e^{\psi} g_{A B}^{\prime}, \square \rho=\rho_{A}^{\mid A}$ and $R^{(2)}$ is the scalar curvature of $g_{A B}$. Given the surface $\Sigma$ equation (6) yields a complicated second order equation for $\psi$. If it is satisfied and

$$
\tilde{R}_{A}^{A} \tilde{R}_{00}>0
$$

then metric (3) is a perfect fluid solution of the Einstein equations.

In this approach the energy density $\epsilon$ and the pressure $p$ of the fluid are given by the following expressions (we omit the constant $8 \pi G / c^{4}$ )

$$
\begin{aligned}
\epsilon & =\frac{1}{2} R_{A}^{A}+\rho^{-1} \square \rho, \\
p & =\frac{1}{2} R_{A}^{A} .
\end{aligned}
$$

If $\square \rho=0$ then $p=\epsilon$. This case is described by a close analogue of the Ernst equation, which admits various solution generating methods [2]. For this reason we will focus here on the case $p \neq \epsilon$.

In order to satisfy the condition $p<\epsilon$ one should require

$$
\gamma \square^{\prime} \rho>0
$$

where $\square^{\prime}$ denotes the wave operator related to the metric $g_{A B}^{\prime}$. If one assumes

$$
\tilde{R}_{A}^{A}>0
$$

in addition to (91) and (12) then the energy dominant condition $\epsilon>|p|$ is satisfied.

In this paper we apply the above approach to surfaces admitting a continuous symmetry. In section 2 we classify surfaces $\Sigma$ invariant under a 1-dimensional group of isometries of $M^{2,1}$. In sections $3-5$ we calculate the corresponding metric tensors and we obtain solutions of equation (6) giving rise to homogeneous cosmological solutions of the Einstein equations.

\section{Invariant Surfaces}

Under translations and Lorentz transformations of coordinates $x^{i}$ of $M^{2,1}$ the induced metric of $\Sigma, g_{I}=d x_{i} d x^{i}$, and its second fundamental form, $g_{I I}=d n_{i} d x^{i}$, remain unchanged. The same is true for metric (3) provided $\psi$ is a scalar function and coordinates $x^{a}$ undergo a linear spinor transformation corresponding to the Lorentz one.

Now, assume that a surface $\Sigma$ is invariant under an active Poincaré transformation $P$ of $M^{2,1}$. Then the forms $g_{I}$ and $g_{I I}$ are preserved. The same 
refers to the metric (3) provided $\psi$ is invariant under $P$ and $x^{a}$ undergo the corresponding spinor transfromation. Thus, the symmetry of $\Sigma$ and $\psi$ induces a symmetry of the 4-dimensional spacetime metric $g$. Note that the invariance of $\psi$ is compatible with equation (6).

In what follows we assume that $\Sigma$ is invariant under a 1-dimensional group of isometries of $M^{2,1}$ corresponding to a Killing vector $k$ (we will call such $\Sigma$ an invariant surface). If $\psi$ is also invariant the metric $g$ acquires a new continuous symmetry in addition to the symmetries $\partial_{a}$.

A general Killing field $k$ in $M^{2,1}$ has the form

$$
k=\left(\epsilon^{i j l} A_{i} x_{j}+B^{l}\right) \partial_{l},
$$

where $\vec{A}, \vec{B}$ are constant vectors, $\epsilon^{i j l}$ is the completely antisymmetric tensor and indices $i, j$ are raised or lowered by means of the 3-dimensional Minkowski metric $\eta_{i j}$. By a Lorentz rotation the vector $\vec{A}$ can be put into one of three canonical forms depending whether it is timelike $(\mathrm{T})$, spacelike $(\mathrm{S})$ or null (N). Then one can shift coordinates $x^{i}$ in order to simplify vector $\vec{B}$. In this way one obtains the following canonical forms of the Killing fields with a nonvanishing rotational part (only such symmetries are admitted by surfaces with a nonvanishing curvature $K$ )

$\mathrm{T}$ :

$$
k=-x_{2} \partial_{1}+x_{1} \partial_{2}+b \partial_{3},
$$

$\mathrm{S}:$

$k=-x_{3} \partial_{2}+x_{2} \partial_{3}+b \partial_{1}$,

$\mathrm{N}$ :

$$
k=\left(x_{1}-x_{3}\right) \partial_{2}+x_{2}\left(\partial_{3}-\partial_{1}\right)+b\left(\partial_{1}+\partial_{3}\right),
$$

where $b$ is a constant.

The Killing field $k$ has to be tangent to the surface $\Sigma$ if the latter is invariant under the 1-parameter group of transformations generated by $k$. Hence, the surface can be defined by an equation $F\left(I_{1}, I_{2}\right)=0$, where $I_{1}, I_{2}$ are two independent solutions of the equation $k^{i} I_{, i}=0$ in $M^{2,1}$. For fields (15)-(17) invariant surfaces with nonzero curvature are given, in some coordinates $x^{i}$, by one of the following sets of relations:

$$
\begin{aligned}
& \mathrm{T}: \quad x^{1}=\tau \cos \varphi, \quad x^{2}=\tau \sin \varphi, \quad x^{3}=a(\tau)+b \varphi, \quad \tau \geq 0, \\
& \mathrm{~S} 1: \quad x^{1}=a(\tau)+b \varphi, \quad x^{2}=\tau \cosh \varphi, \quad x^{3}=\tau \sinh \varphi, \quad \tau \geq 0, \\
& \mathrm{~S} 2: \quad x^{1}=a(\tau)+b \varphi, \quad x^{2}=\tau \sinh \varphi, \quad x^{3}=\tau \cosh \varphi, \quad \tau \geq 0 \text {, } \\
& \mathrm{N} 1: \frac{v^{2}}{2 b}-2 x_{2}=a\left(u+\frac{v x_{2}}{b}-\frac{v^{3}}{6 b^{2}}\right), \quad x^{1}=\frac{1}{2}(v+u), \quad x^{3}=\frac{1}{2}(v-u) \\
& \mathrm{N} 2: u+\frac{x_{2}{ }^{2}}{v}=a(v), \quad x^{1}=\frac{1}{2}(v+u), \quad x^{3}=\frac{1}{2}(v-u) \\
& \mathrm{N} 3: u+\frac{v x_{2}}{b}-\frac{v^{3}}{6 b^{2}}=\text { const }, \quad x^{1}=\frac{1}{2}(v+u), \quad x^{3}=\frac{1}{2}(v-u),
\end{aligned}
$$


where $a$ is an arbitrary function of one variable and $b \neq 0$ in the cases N1 and N3.

In sections 3-5 we derive metrics corresponding to surfaces (18)-(23).

\section{Metrics related to surfaces of type $\mathbf{T}$}

Due to a residual freedom in choice of coordinates $x^{i}$ for surfaces (18) one can assume

$$
b \geq 0
$$

without a loss of generality. In order to guarantee the spacelike character of $\Sigma$ the function $a(\tau)$ should satisfy

$$
\dot{a}^{2}<1-b^{2} \tau^{-2},
$$

where the dot denotes the derivative with respect to $\tau$. The normal vector satisfying condition (2) reads

$$
\vec{n}=\frac{1}{\sqrt{\tau^{2}\left(1-\dot{a}^{2}\right)-b^{2}}}(\tau \dot{a} \cos \varphi-b \sin \varphi, \tau \dot{a} \sin \varphi+b \cos \varphi,-\tau) .
$$

The second fundamental form is given by

$$
g_{I I}=\frac{1}{\sqrt{\tau^{2}\left(1-\dot{a}^{2}\right)-b^{2}}}\left(\tau \ddot{a} d \tau^{2}-2 b d \tau d \varphi+\tau^{2} \dot{a} d \varphi^{2}\right) .
$$

The Gauss curvature $K=\operatorname{det} g_{I I} / \operatorname{det} g_{I}$ is negative provided

$$
\dot{a} \ddot{a}<b^{2} \tau^{-3} .
$$

Under conditions (25) and (28) definition (41) yields

$$
\rho=\frac{\tau^{2}\left(1-\dot{a}^{2}\right)-b^{2}}{\sqrt{b^{2}-\tau^{3} \dot{a} \ddot{a}}} .
$$

In order to describe the corresponding spacetime metric (3) it is convenient to use coordinates $\tau, x, y, z$, where $x^{a}=x, y$ and

$$
z=\frac{1}{2}(\varphi-\sigma), \quad \sigma(\tau)=b \int \frac{d \tau}{\tau^{2} \dot{a}} .
$$

In these coordinates the metric takes the following form

$$
\begin{aligned}
g= & \gamma e^{\psi}\left[\left(\frac{b^{2}}{4 \tau^{4} \dot{a}^{2}}-\frac{\ddot{a}}{4 \tau \dot{a}}\right) d \tau^{2}-d z^{2}\right]-\sqrt{\frac{\tau^{2}\left(1-\dot{a}^{2}\right)-b^{2}}{b^{2}-\tau^{3} \dot{a} \ddot{a}}}[(\tau-\tau \dot{a} \cos \sigma+b \sin \sigma) \\
& \left.\left(\theta^{2}\right)^{2}-2(\tau \dot{a} \sin \sigma+b \cos \sigma) \theta^{2} \theta^{3}+(\tau+\tau \dot{a} \cos \sigma-b \sin \sigma)\left(\theta^{3}\right)^{2}\right]
\end{aligned}
$$


where

$$
\theta^{2}=\cos z d x+\sin z d y, \quad \theta^{3}=-\sin z d x+\cos z d y
$$

The Einstein equations for metric (31) reduce to equation (6) and condition (91).

Under the assumption

$$
\gamma=1, \psi=\psi(\tau)
$$

metric (31) is a cosmological solution of Bianchi type $V I I_{0}$. In the generic case the fluid velocity is not orthogonal to the surfaces of homogeneity $\tau=$ const (so called tilted solution). Since metric (31) depends on two functions $a(\tau), \psi(\tau)$ one can impose on $p$ and $\epsilon$ a barotropic equation of state

$$
p=p(\epsilon) \text {. }
$$

Equations (6), (34) together with condition (9) and, if required, conditions (12), (13) compose a system of equations for functions $a(\tau)$ and $\psi(\tau)$.

When (33) is satisfied and

$$
b=0
$$

the fluid velocity is orthogonal to the surfaces $\tau=$ const. Then equation (6) reduces to

$$
\tilde{R}_{11}=0
$$

and inequality (9) becomes a part of the energy condition (13). Equation (36) yields

$$
\psi=\log \rho-\int \frac{\left(a-a_{0}\right) \ddot{a}}{\tau\left(1-\dot{a}^{2}\right)} d \tau,
$$

where $a_{0}$ is a constant and

$$
\rho=\left(1-\dot{a}^{2}\right) \sqrt{-\frac{\tau}{\dot{a} \ddot{a}}}
$$

(note that $|\dot{a}|<1, \dot{a} \ddot{a}<0$ due to (25) and (28)). The corresponding spacetime metric reads

$$
\begin{aligned}
g= & \rho e^{\omega}\left(-\frac{\ddot{a}}{4 \tau \dot{a}} d \tau^{2}-d z^{2}\right)-\rho \sqrt{\frac{1-\dot{a}}{1+\dot{a}}}(\cos z d x+\sin z d y)^{2}+ \\
& -\rho \sqrt{\frac{1+\dot{a}}{1-\dot{a}}}(-\sin z d x+\cos z d y)^{2}
\end{aligned}
$$


where function $\omega(\tau)$ is given by

$$
\omega=-\int \frac{\left(a-a_{0}\right) \ddot{a}}{\tau\left(1-\dot{a}^{2}\right)} d \tau .
$$

In this case expressions (10) and (11) for the fluid energy and pressure yield

$$
\begin{aligned}
& \epsilon(\tau)=\rho^{-2} e^{-\omega}\left(\frac{3 \rho^{\prime 2}}{4 \rho}+\frac{\lambda \rho^{\prime}}{\rho}\left(a-a_{0}\right)-\frac{\lambda a^{\prime}}{2}-\frac{\tau^{2}}{\rho}\right), \\
& p(\tau)=\epsilon(\tau)-\frac{\rho^{\prime \prime}}{\rho^{2}} e^{-\omega} .
\end{aligned}
$$

Here $\lambda=\operatorname{sgn}(\dot{a})$ and we denote

$$
f^{\prime}=\dot{f} \sqrt{\frac{4 \tau|\dot{a}|}{|\ddot{a}|}}
$$

for any function $f(\tau)$. Expressions (41), (42) contain one arbitrary function $a(\tau)$, which can be fitted in order to satisfy a barotropic equation of state (34) of the fluid. Due to the complicated structure of (41) and (42) this fitting probably requires numerical calculations. On the other hand it is not difficult to find examples of $a(\tau)$ for which the energy dominant condition is satisfied. Such example was given in 6 . In this example the asymptotic behaviour of $p$ and $\epsilon$ is similar to that in the model of Demiański and Grishschuk [7.

\section{Metrics related to surfaces of type $S$}

In this section we consider metrics related to the invariant surfaces (19) and (201). In both cases the second fundamental form is proportional to

$$
g_{A B}^{\prime} d x^{A} d x^{B}=\frac{\ddot{a}}{4 \tau \dot{a}} d \tau^{2}-\frac{b}{2 \tau^{2} \dot{a}} d \tau d \varphi-\frac{1}{4} d \varphi^{2},
$$

however the corresponding spacetime metrics are different. The Gauss curvature $K$ is negative provided

$$
\dot{a} \ddot{a}>-b^{2} \tau^{-3} .
$$

The surface (19) is spacelike iff

$$
\dot{a}^{2}<-1+b^{2} \tau^{-2} .
$$

Since $b \neq 0$ one can assume

$$
b>0
$$


without a loss of generality. The function $\rho$ is given by

$$
\rho=\frac{b^{2}-\tau^{2}\left(1+\dot{a}^{2}\right)}{\sqrt{b^{2}+\tau^{3} \dot{a} \ddot{a}}} .
$$

The corresponding spacetime metric (3) can be transformed to the form

$$
g=\gamma e^{\psi}\left[\left(\frac{b^{2}}{4 \tau^{4} \dot{a}^{2}}+\frac{\ddot{a}}{4 \tau \dot{a}}\right) d \tau^{2}-d z^{2}\right]-\sqrt{\frac{b^{2}-\tau^{2}\left(1+\dot{a}^{2}\right)}{b^{2}+\tau^{3} \dot{a} \ddot{a}}}[(-\tau+\tau \dot{a} \sinh \sigma+
$$

$\left.b \cosh \sigma)\left(\theta^{2}\right)^{2}+2(\tau \dot{a} \cosh \sigma+b \sinh \sigma) \theta^{2} \theta^{3}+(\tau+\tau \dot{a} \sinh \sigma+b \cosh \sigma)\left(\theta^{3}\right)^{2}\right]$,

where

$$
\begin{aligned}
& \sigma(\tau)=b \int \frac{d \tau}{\tau^{2} \dot{a}}, \\
& \theta^{2}=\cosh z d x-\sinh z d y, \quad \theta^{3}=-\sinh z d x+\cosh z d y
\end{aligned}
$$

and the new coordinate $z$ is related to $\varphi$ via

$$
z=\frac{1}{2}(\varphi+\sigma)
$$

For the surface (20) the function $a(\tau)$ has to obey condition (45) and

$$
\dot{a}^{2}>1+b^{2} \tau^{-2} .
$$

Due to a residual freedom of coordinates $x^{i}$ one can replace condition (53) by

$$
\dot{a}>\sqrt{1+b^{2} \tau^{-2}}
$$

without a loss of generality. In this case

$$
\rho=\frac{\tau^{2}\left(\dot{a}^{2}-1\right)-b^{2}}{\sqrt{b^{2}+\tau^{3} \dot{a} \ddot{a}}}
$$

and the corresponding spacetime metric is given by

$$
\begin{gathered}
g=\gamma e^{\psi}\left[\left(\frac{b^{2}}{4 \tau^{4} \dot{a}^{2}}+\frac{\ddot{a}}{4 \tau \dot{a}}\right) d \tau^{2}-d z^{2}\right]-\sqrt{\frac{\tau^{2}\left(\dot{a}^{2}-1\right)-b^{2}}{b^{2}+\tau^{3} \dot{a} \ddot{a}}}[(-\tau+\tau \dot{a} \cosh \sigma+ \\
\left.b \sinh \sigma)\left(\theta^{2}\right)^{2}+2(\tau \dot{a} \sinh \sigma+b \cosh \sigma) \theta^{2} \theta^{3}+(\tau+\tau \dot{a} \cosh \sigma+b \sinh \sigma)\left(\theta^{3}\right)^{2}\right]
\end{gathered}
$$

and relations (501)-(52). 
In both cases (49) and (56) the function $\psi$ has to satisfy equation (6) and condition (91). If $\gamma=1$ and $\psi=\psi(\tau)$ these metrics are cosmological solutions of Bianchi type $V I_{0}$ (tilted if $\left.b \neq 0\right)$.

If $\gamma=1, \psi=\psi(\tau)$ and $b=0$ the metric (56) takes the form

$$
\begin{aligned}
g= & \rho e^{\omega}\left(\frac{\ddot{a}}{4 \tau \dot{a}} d \tau^{2}-d z^{2}\right)-\rho \sqrt{\frac{\dot{a}-1}{\dot{a}+1}}(\cosh z d x-\sinh z d y)^{2}+ \\
& -\rho \sqrt{\frac{\dot{a}+1}{\dot{a}-1}}(-\sinh z d x+\cosh z d y)^{2},
\end{aligned}
$$

where

$$
\rho=\left(\dot{a}^{2}-1\right) \sqrt{\frac{\tau}{\dot{a} \ddot{a}}}
$$

and the function $a$ is assumed to satisfy $\dot{a}>1, \ddot{a}>0$ (see (54) and (45)). In this case the general solution of (6) is given by (40) and the fluid energy density and pressure are given by (41)-(43).

\section{Metrics related to surfaces of type $\mathrm{N}$}

In order to parametrize the surface (21) it is convenient to use coordinates $\tau$ and $z$ given by

$$
\begin{aligned}
& \tau=u+\frac{v x_{2}}{b}-\frac{v^{3}}{6 b^{2}} \\
& z=v+\sigma, \quad \sigma(\tau)=\frac{1}{2} \int \dot{a}^{2} d \tau
\end{aligned}
$$

The surface is spacelike and $K<0$ iff

$$
b^{-1} a \dot{a}^{2}>2, \quad \dot{a}^{4}+4 b \ddot{a}>0 .
$$

Under conditions (61) definition (41) yields

$$
\rho=\frac{\left|a \dot{a}^{2}-2 b\right|}{\sqrt{\dot{a}^{4}+4 b \ddot{a}}} .
$$

It follows from (61) that $\dot{a} \neq 0$. Hence, one can assume

$$
\dot{a}>0
$$


without a loss of generality. Let $2 b x, y$ be the coordinates $x^{a}$ in the expression (3) for the spacetime metric. In the coordinates $\tau, x, y, z$ this metric reads

$$
\begin{aligned}
g= & \gamma e^{\psi}\left[\left(\frac{\dot{a}^{4}}{4}+b \ddot{a}\right) d \tau^{2}-d z^{2}\right]-\rho \sqrt{\frac{2}{b^{-1} a \dot{a}^{2}-2}}\left[\dot{a}(d y-z d x)^{2}+\right. \\
& \left.+(2 \dot{a} \sigma-4 b)(d y-z d x) d x+\left(\dot{a} \sigma^{2}-4 b \sigma+2 b a \dot{a}\right) d x^{2}\right] .
\end{aligned}
$$

The Einstein equations reduce to equation (6) and condition (9). If $\gamma=1$ and $\psi=\psi(\tau)$ the metric (64) is a tilted cosmological solution of Bianchi type $I I$.

The surface (22) is preserved by the Killing vector field (17) with $b=0$. It is convenient to parametrize $\Sigma$ in the following way

$$
x^{i}(\tau, z)=\frac{1}{2}\left(\left(1-z^{2}\right) \tau+a(\tau), 2 \tau z,\left(1+z^{2}\right) \tau-a(\tau)\right), \quad \tau \geq 0 .
$$

The surface is spacelike and $K<0$ iff

$$
\dot{a}>0, \quad \ddot{a}>0 .
$$

In this case

$$
\rho=\dot{a} \sqrt{\frac{2 \tau}{\ddot{a}}}
$$

and the corresponding spacetime metric reads

$$
g=\gamma e^{\psi}\left(\frac{\ddot{a}}{2 \tau} d \tau^{2}-d z^{2}\right)-\dot{a} \sqrt{\frac{2 \tau}{\ddot{a}}}\left[\sqrt{\dot{a}} d x^{2}+\frac{1}{\sqrt{\dot{a}}}(d y-z d x)^{2}\right] .
$$

The function $\psi$ has to satisfy equation (6) and condition (91).

If $\gamma=1$ and $\psi=\psi(\tau)$ the metric (68) is a non-tilted cosmological solution of Bianchi type $I I$. It reads

$$
g=\rho \sqrt{\dot{a}} e^{\omega}\left(\frac{\ddot{a}}{2 \tau} d \tau^{2}-d z^{2}\right)-\sqrt{\frac{2 \tau \dot{a}}{\ddot{a}}}\left[\dot{a} d x^{2}+(d y-z d x)^{2}\right],
$$

where

$$
\omega(\tau)=\omega_{0} \int \frac{\ddot{a}}{\tau \dot{a}}, \quad \omega_{0}=\text { const } .
$$

The corresponding energy density and pressure of the fluid can be expressed in the following way

$$
\begin{aligned}
& \epsilon(\tau)=\frac{e^{-\omega}}{\rho^{2} \sqrt{\dot{a}}}\left(\frac{3 \rho^{\prime 2}}{4 \rho}+\frac{\rho^{\prime}}{2 \rho}\left(\tau+2 \omega_{0}\right)-\frac{\tau^{\prime}}{4}-\frac{\tau^{2}}{4 \rho}\right), \\
& p(\tau)=\epsilon-\frac{\rho^{\prime \prime}}{\sqrt{\dot{a}} \rho^{2}} e^{-\omega}
\end{aligned}
$$


where we denote

$$
f^{\prime}=\dot{f} \sqrt{\frac{2 \tau}{\ddot{a}}}
$$

for any function $f(\tau)$. Metrics (69) include particular solutions of Collins and Stewart [8] ( metrics (14.20) in [2]) which satisfy $p=(\gamma-1) \epsilon$ with a constant $\gamma$. They follow from (69) for $a=\frac{1}{n} \tau^{n}$ where $n=\frac{2+\gamma}{4-2 \gamma}$ and $\frac{2}{3}<\gamma<2$.

The surface (23) induces the following spacetime metric

$$
g=2 \gamma e^{\psi} d \tau d z-\tau^{3} d x^{2}-\tau(d y-z d x)^{2}
$$

in some coordinates $\tau, x, y, z$. In this case the function $\psi$ is given by

$$
\psi(\tau, z)=\frac{1}{4} \log \tau-\int \frac{(z+c(\tau))^{2}}{4 \tau^{3}} d \tau
$$

where $c(\tau)$ is an arbitrary function. Metric (174) is not interesting because it yields $p=\epsilon<0$.

\section{Summary}

Following the method elaborated in [6] we have constructed several classes of perfect fluid solutions of the Einstein equations. The solutions are related to spacelike surfaces with a negative curvature in the 3-dimensional Minkowski space $M^{2,1}$. We classified those surfaces which are invariant under a 1-dimensional group of isometries of $M^{2,1}$ (equations (18)-(23)). For all of them we calculated the corresponding spacetime metrics (equations (31), (49), (56), (64), (68), (74)). In all cases except (74) they depend on two functions $a, \psi$ and the Einstein equations reduce to equation (6) and inequality (92). Under assumption (33) these metrics become cosmological solutions of Bianchi type $I I, V I_{0}$ or $V I I_{0}$ and a barotropic equation of state can be imposed. In some cases (see (39), (57) and (69)) we succeded to solve all of the Einstein equations up to quadratures. The resulting metrics depend on an arbitrary function of time, which can be further specified in order to satisfy an equation of state.

Acknowledgments. This work was partially supported by the Polish Committee for Scientific Research (grant 2 P03B 12724 and 2 P03B 036 23). 


\section{References}

[1] A. Krasiński, Inhomogeneous cosmological models, Cambridge University Press 1997.

[2] H. Stephani, D. Kramer, , M. MacCallum, C. Hoenselaers and E. Herlt, Exact Solutions of Einstein's Field Equations Second Edition, Cambridge University Press 2003.

[3] J.M.M. Senovilla, New class of inhomogeneous cosmological perfect fluid solutions without Big Bang singularity, Phys. Rev. Lett. 64 (1990), 2219.

[4] E. Ruiz and J.M.M. Senovilla, General class of inhomogeneous perfect fluid solutions, Phys. Rev. D 64 (1992), 1995.

[5] M. Mars and J.M.M. Senovilla, Non-diagonal $G_{2}$ separable perfect fluid spacetimes, Class. Quantum Grav. 14 (1997), 205.

[6] A.Szereszewski and J. Tafel, Perfect Fluid Spacetimes With Two Symmetries, paper sent to Class. Quantum Grav.

[7] M. Demiański and L.P. Grishschuk, Homogeneous rotating universe with flat space, Commun. Math. Phys. 25 (1972), 233.

[8] C.B. Collins and J.M. Stewart, Qualitative cosmology, Mon. Not. R. Astron. Soc. 153 (1971), 419. 